\title{
A Boundary Collocation Method for Wave Propagation through Obstacle
}

\author{
Xueling $\mathrm{CaO}^{1,2, \mathrm{a}}$, Yang $\mathrm{Liu}^{1,2, \mathrm{~b}}$, Zan Huang ${ }^{1, \mathrm{c}}, \mathrm{R}^{\mathrm{Ri}} \mathrm{Li}^{1}$, Junjie $\mathrm{Tong}^{1}$ \\ ${ }^{1}$ GuangZhou maritime university, Guangzhou 510725, China; \\ ${ }^{2}$ GuangZhou Institute of Energy Conversion, Chinese Academy of Sciences, Guangzhou 510640, \\ China; \\ accaoxueling@126.com, byangliu@ms.ustc.ac.cn, chuangzan@gzhmt.edu.cn
}

Keywords: boundary collocation method; eigenfunction expansion method; wave propagation; obstacle

Abstract. The interaction between wave and the obstacle in water is a common problem in ocean engineering. The article use a new numerical method, Boundary Collocation Method (BCM) based on Eigenfunction Expansion Method (EEM), to solve the two-dimensional wave problems between wave and obstacle. The obstacle is no longer confined to the rectangle, but the submersed wedge obstacle. The change of the wave velocity potential will be analysed.

\section{Introduction}

The study of wave propagation through obstacles is of considerable importance for wave power device and coastal engineering. Many studies have been devoted to wave propagation theories based on analytic and numerical methods (Stokes, 1947 ${ }^{[1]}$; Wen, 1964a ${ }^{[2]}, 1964 \mathrm{~b}^{[3]}$; Alliney, 1981 ${ }^{[4]}$; Aimi $^{[5]}$ et al., 2011). As Stoker(1957) ${ }^{[6]}$ pointed out, during the wave propagation through obstacle, the wave's potential function will have a singularity to make the amplitude tend to infinity. So it is particularly complex.

Generally the analytic and numerical methods are available for solving the wave propagation. However, both methods have their advantages and disadvantages. The analytic method can solve the problems only with several simple boundaries (Wu et al., 1995 ${ }^{[7]}$; Zheng et al., 2004 ${ }^{[8]}$ ). The numerical methods, particularly the hybrid method of Boundary Element Method (BEM) and EEM have been widely used for the numerical solutions of potential problems in recent years. In the case of small wave problem, BEM can be used to express the solution of the Laplace equation by boundary conditions and boundary discretization (Alliney, $1981^{[4]}$ ). It brings difficulties in solving practical problems in ocean engineering.

The attraction of BCM lies on its applicability to the problems with irregular domains and arbitrary boundary conditions, its ease of programming, and its conceptual simplicity. Applications of BCM for the analysis of plates and shells can be found in the paper by Hutchinson(1991 $)^{[9]}$ and Zheng et al. (2007) ${ }^{[10]}$. Previously it is applied to various elastic problems and heat transfer problems (Ramachandran and Gunjal, 2009 ${ }^{[11]}$ ). Reichel and Chapman(1986) ${ }^{[12]}$ apply this idea to edge wave problems.

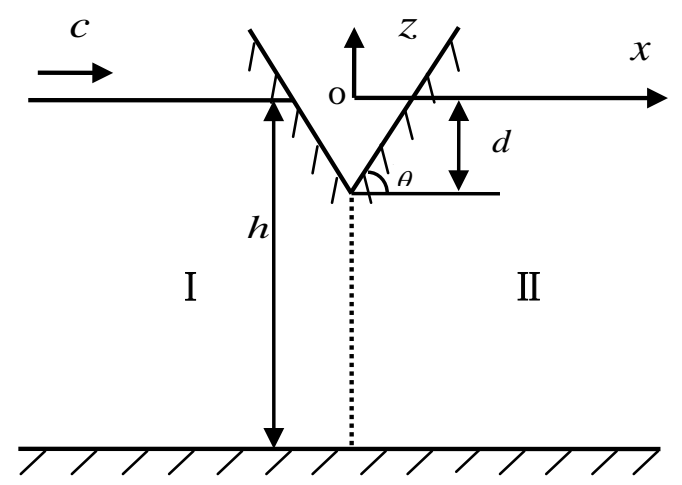

Fig. 1. The Physical model. 
In this paper, to avoid the complexity of the calculation and solve problems of submersed wedge obstacle, a hybrid method of BCM and EEM is used.

\section{Mathematical Model}

Considering a submersed wedge obstacle fixed on the surface, Fig. 1 shows the physical model and the Cartesian coordinate system. The negative $x$-axis directs right to the sea, while the positive $Z$-axis takes vertically upward from the water surface. Here $h$ denotes the depth of the sea, and $d$ is the draft of the obstacle. The obstacle boundary can be expressed as a function $f(x)$. The income wave propagate through obstacle from region I to region II with velocity $c$ (here area I and area II are divided by dotted line in Fig. 1).

Assuming the flow motion is irrotational, the waves are of small amplitude and the fluid is incompressible and inviscid. $\Phi(x, z, t)$ defines the velocity $v=\nabla \Phi$. For simple waves, any point moves at a fixed frequency $\omega$. The changes of $\Phi(x, z, t)$ to time $t$ should be presented in harmonic forms $\Phi(x, z, t)=\operatorname{Re}\left\{\phi(x, z) \mathrm{e}^{\mathrm{i} \omega t}\right\}$.

Where, $\phi(x, z)$ is a complex potential function independent of time. The wave motion has to satisfy conditions at the obstacle boundaries, free surface and the seabed, respectively.

$$
\left\{\begin{array}{l}
\nabla^{2} \phi=0 \\
\left.\left(\frac{\partial \phi}{\partial z}-\frac{\omega^{2}}{\mathrm{~g}} \phi\right)\right|_{z=0}=0 \\
\left.\frac{\partial \phi}{\partial n}\right|_{z=-h}=0 \\
\left.\frac{\partial \phi}{\partial n}\right|_{z=f(\mathrm{x})}=0
\end{array}\right.
$$

Eq. (2) is the linear wave free surface boundary condition. Instead of the actual free surface, it applied to the undisturbed surface. Eq. (3) is the boundary condition on the impermeable and immovable seabed. The obstacle boundary can be set as $f(x)=\left\{\begin{array}{l}-x \tan \theta-d(x<0) \\ x \tan \theta-d(x>0)\end{array}\right.$. In addition, we have a regularity condition at the negative infinity: $\lim _{x \rightarrow-\infty}|\phi(x, z)|<+\infty, z \in[-\mathrm{h}, 0]$.

\section{Solution to Potential}

\subsection{Expression for Potential}

To find a method widely used in wave problems, the function $\phi(x, z)$ satisfying the bottom condition Eq. (3) and the free surface condition Eq. (2) has been given by Alliney (1981) ${ }^{[4]}$ as follows:

$$
\phi=\mathrm{A}\left\{\cosh \left[k_{0}(z+\mathrm{h})\right] \mathrm{e}^{\mathrm{i} k_{0} x}+a_{0} \cosh \left[k_{0}(z+\mathrm{h})\right] \mathrm{e}^{-i k_{0} x}\right\}+\sum_{j=1}^{+\infty} a_{j} \cos \left[k_{j}(z+\mathrm{h})\right] \mathrm{e}^{k_{j} x}+\sum_{j=1}^{+\infty} b_{j} \cos \left[k_{j}(z+\mathrm{h})\right] \mathrm{e}^{-k_{j} x}
$$

Where, $a_{0}, a_{j}$ and $b_{j}(j=1,2 \cdots n)$ are complex constants. By considering the infinity condition, take $b_{j}=0$ for any $j \cdot k_{0}$ and $k_{j}(j=1,2 \cdots n)$ follow the relations: $\frac{\omega^{2}}{\mathrm{~g}}=k_{0} \tanh \left(k_{0} \mathrm{~h}\right)$ and $\frac{\omega^{2}}{\mathrm{~g}}=-k_{j} \tan \left(k_{j} \mathrm{~h}\right)$.

For simplicity, the constant $\mathrm{A}$ can be set as $\mathrm{A}=\frac{1}{\cosh \left(k_{0} \mathrm{~h}\right)}$.

Then to this wave problem in region I and II , Eq. (5) can be written as:

$$
\phi_{R 1}(x, z)=\left(\mathrm{e}^{\mathrm{i} k_{0} x}+a_{0} \mathrm{e}^{-\mathrm{i} k_{0} x}\right) \frac{\cosh \left(k_{0}(z+h)\right)}{\cosh \left(k_{0} h\right)}+\sum_{j=1}^{+\infty} a_{j} \mathrm{e}^{k_{j} x} \cos \left(k_{j}(z+h)\right)
$$




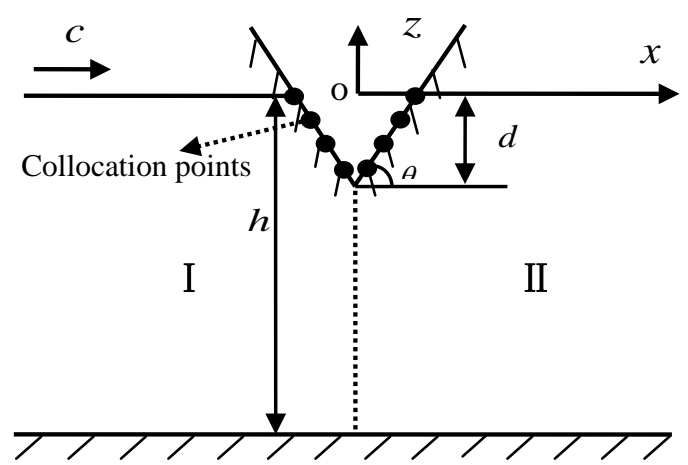

Fig. 2 Collocation points on barrier boundary

$\phi_{R 2}(x, z)=b_{0} \mathrm{e}^{\mathrm{i} k_{0} x} \cosh \left[k_{0}(z+h)\right]+\sum_{j=1}^{+\infty} b_{j} \mathrm{e}^{-k_{j} x} \cos \left[k_{j}(z+h)\right]$

Eq. (6) and Eq.(7), the analytic solution satisfying the bottom condition and the free surface condition, is the basis equation. To obstacle boundary, the BCM will be used to obtain more accurate results.

\subsection{BCM for the Unknown Coefficients}

Select the appropriate points on boundary, which satisfy the condition at $x<0$ and $x>0$. $\phi_{R 1}{ }^{\prime}(x, z)$ with $j=N_{1}$ and $\phi_{R 2}{ }^{\prime}(x, z)$ with $j=N_{2}$ denotes the numerical result of the potential. To determine the $N_{1}+N_{2}+2$ unknown coefficients, considering the continuity conditions:
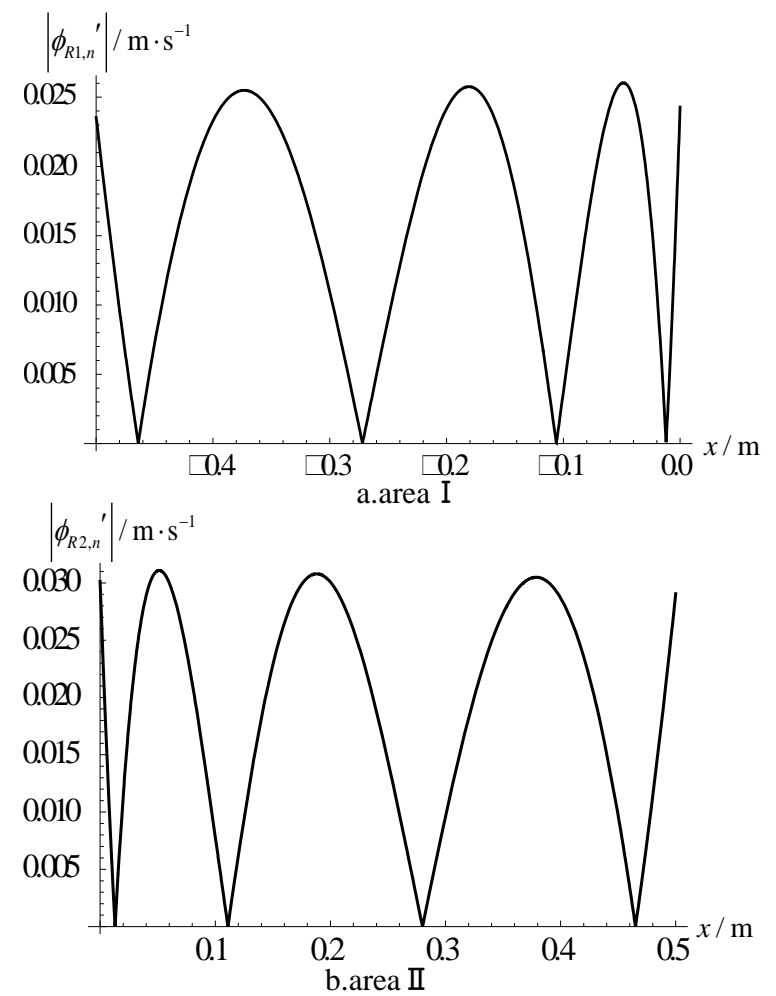

Fig. 3 The normal velocity on barrier boundary ( $d=2$, Projection to the $\mathrm{x}$ axis)

1) the continuity condition of normal velocity in $x=0: \frac{\partial \phi_{R 1}}{\partial x}=\frac{\partial \phi_{R 2}}{\partial x},(x=0,-h<z<-d)$

2) the continuity condition of pressure in $x=0: \phi_{R 1}=\phi_{R 2},(x=0,-h<z<-d)$ 
Take $M_{1}\left(M_{1}<N_{1}\right)$ points in region I and $M_{2}\left(M_{2}<N_{2}\right)$ points in region II, it will be $M_{1}+M_{2}$ linear equations to obtain $M_{1}+M_{2}$ coefficients. To the other coefficients, $N_{1}+N_{2}-M_{1}-M_{2}+2$ adjusted points are set on obstacle (Fig. 2).

All of the coefficients will be obtained from the linear equations. But the obstacle boundary condition Eq.(4) is only satisfied at $N_{1}+N_{2}-M_{1}-M_{2}+2$ chosen points. There must be errors on obstacle.
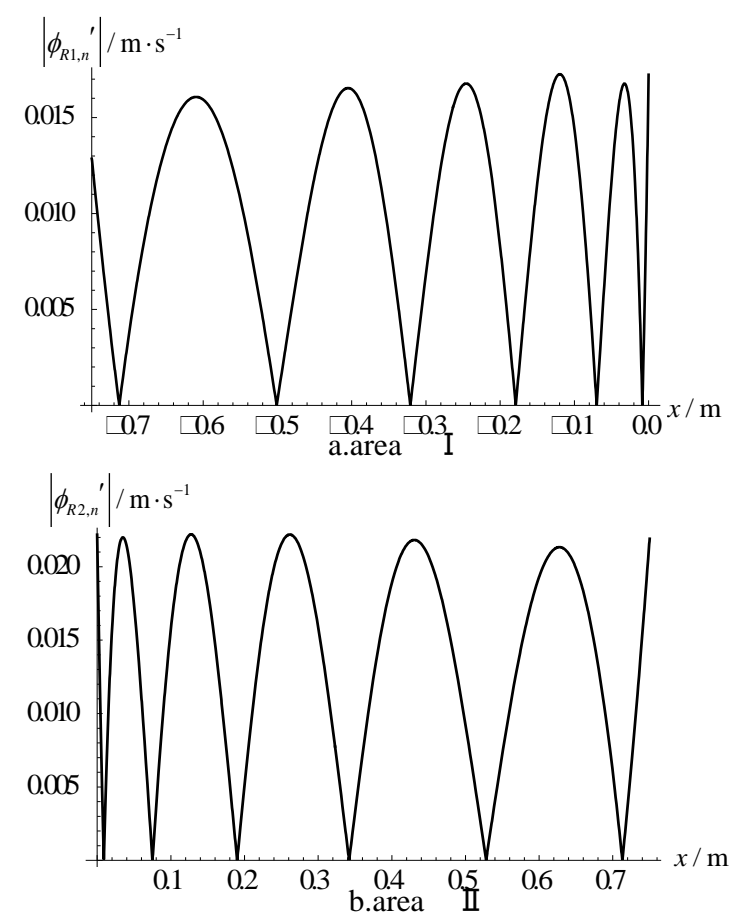

Fig. 4 The normal velocity on barrier boundary ( $d=3$, Projection to the $\mathrm{x}$ axis)

\section{Numerical results and discussion}

\subsection{Numerical results}

Set $h=10 \mathrm{~m}, \omega=1.25 \mathrm{rad} / \mathrm{s}, \tan \theta=4$, and obstacle boundary $F(x)=\left\{\begin{array}{l}-4 x-d(x<0) \\ 4 x-d(x>0)\end{array}\right.$. Set $d=2$ and $N_{1}=N_{2}=11, M_{1}=M_{2}=8$; the value of $j$ from 1 to 11 and $i^{\prime}$ from 1 to 8 . So 4 points can be set in region I and 4 points in region II to obtain the coefficients. Fig. 3 shows the absolute error $\left|\phi_{R 1, n}^{\prime}\right|$ and $\left|\phi_{R 2, n}^{\prime}\right|$.

From Fig.3, the maximum absolute error is no more than 0.025 in region I and 0.03 in region II , which have a high accuracy.

While, set $d=3, \quad N_{1}=N_{2}=13, M_{1}=M_{2}=8$, and $j$ value from 1 to $13, i^{\prime}$ from 1 to 8 . So 6 points can be set in region I and 6 points in region II. Fig. 4 shows the absolute error $\left|\phi_{R 1, n}^{\prime}\right|$ and $\left|\phi_{R 2, n}^{\prime}\right|$.

From Fig.4, the maximum absolute error is no more than 0.017 in region I and 0.022 in region II, which have a higher accuracy. Fig. 5 shows the velocity potential on free surface. From the curve,wave propagate from region I to II through the obstacle will lead a amplitude reduction because of the reflected wave generated by obstacle. The deeper the obstacle, the larger the reduction. 

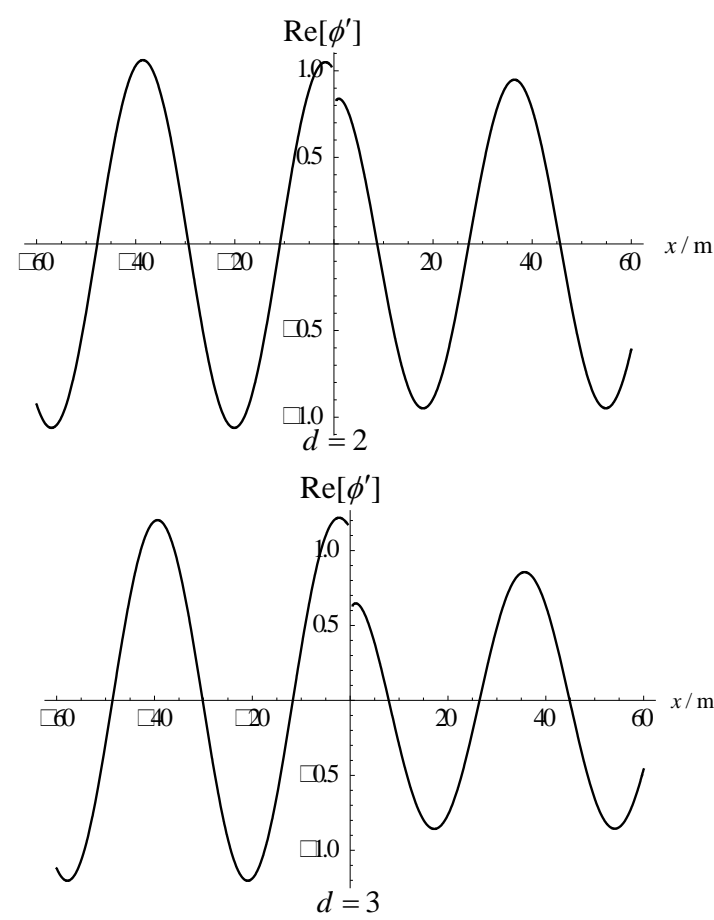

Fig. 5 The velocity potential on free surface $\mathrm{z}=0$

\subsection{Feasibility Analysis}

To verify its feasibility, the relative error are discussed. The relative error is $\varepsilon_{r}=\frac{\left|\phi_{R, n}{ }^{\prime}-\phi_{R, n}\right|}{\left|\phi_{I, n}\right|+1}=\frac{\left|\phi_{n}{ }^{\prime}\right|}{\left|\phi_{I, n}\right|+1}$. When $d=2$, the relative error is no more than 0.02 in region I and 0.025 in region II. When $d=3$, the relative error is no more than 0.014 in region I and 0.017 in region II . It proves the feasibility of the method.

\section{Conclusions}

The application of the hybrid method of BCM and EEM to wave problems proves its validity. Finding the position of the collocation points on obstacle is the key and difficulty of the method. As long as collocating appropriate points, the solutions can be obtained. The study provides the theory basis and feasible measure of the hybrid method for solving the more complicated 2D wave problems. It can be used in not only wedge obstacle, but also arbitrary obstacle.

\section{References}

[1]. Stokes, G.G., 1947. On the theory of oscillatory waves, Trans. Camb. Phil. Soc., 8,441 473.

[2]. Wen, S. C., 1964a. Transformation and braking of waves on sloping beaches (Part 1), Journal of Shandong College of Oceanology, (1):13 30. (in Chinese)

[3]. Wen, S. C., 1964b. Transformation and braking of waves on sloping beaches (Part 2), Journal of Shandong College of Oceanology, (1):31 50. (in Chinese)

[4]. Alliney, S., 1981. A numerical study of water waves on sloping beaches, Appl. Math. Model., 5(5):321 328.

[5]. Aimi, M., Diligenti, M. and Guardasoni, C., 2011. On the energentic Galerkin boundary element method applied to interior wave propagation problems, J. Comput. Appl. Math., 235(7):1746 1754.

[6]. Stoker, J. J., 1957. Water Waves, New York \& London, Interscience Publishers. 
[7]. Wu, C., Watanabe, E. and Utsunomiya, T. 1995. An eigenfunction expansion-matching method for analyzing the wave-induced responses of an elastic floating plate, Appl. Ocean Res., 17(5):301 310.

[8]. Zheng, Y. H., You, Y. G. and Shen, Y. M., 2004. On the radiation and diffraction of water waves by a rectangular buoy, Ocean Eng., 31(8):1063 1082.

[9]. Hutchinson, J. R., 1991. Analysis of plates and shells by boundary collocation, in: Boundary Element Analysis of Plates and Shells, Berlin, Springer. 341 368.

[10]. Zheng, X. P., Huang, Q. Z. and Wang, B., 2007. An adaptive boundary collocation method for plate bending problems, Tsinghua Science and Technology, 12(5):567 571.

[11]. Ramachandran, P. A. and Gunjal, P. R., 2009. Comparison of boundary collocation methods for singular and non-singular axisymmetric heat transfer problems, Eng. Anal. Bound. Elem., 33(5):704 716.

[12]. Reichel, L. and Chapman, P., 1986. Edge waves by boundary collocation, J. Comput. Appl. Math., 15(1):59 73. 